\title{
Bell's phenomenon in newborns and premature babies
}

\author{
M Snir, I Kremer, A Kuperman, P Merlob, Y Yassur
}

\begin{abstract}
Aims-To evaluate the development and course of Bell's phenomenon (BP) in premature and normal neonates.

Methods-Twenty seven preterm infants and 42 healthy full term infants were studied. Mean birth weight, gestational age, and head circumference were recorded. BP was graded from no response to full response. The premature group was examined in the first week of life, and then at 4 and 8 weeks after birth. The full term group was initially examined at the age of 3 days until a full response was observed. Results-No preterm infants exhibited BP during the first week of life, while $8 / 28$ (29.6\%) exhibited a weak to full reflex at 8 weeks of age. In the full term group 15/42 neonates $(35.7 \%)$ demonstrated a mature reflex at age of 3 days and $36 / 37(97.3 \%)$ at age 16 weeks. A significant correlation was observed between BP, sex, and birth weight, as well as gestational age in the premature group. No such correlation was found in the full term infants.

Conclusion-BP has a longitudinal course. It is an important clinical marker for evaluation and follow up of neuroophthalmic maturation in neonates.

(Br f Ophthalmol 1996;80:553-555)
\end{abstract}

Department of

Ophthalmology, Petah

Tiqva, and Sackler

Faculty of Medicine,

Tel Aviv University, Tel

Aviv, Israel

M Snir

I Kremer

YYassur

Neonatal Unit,

Beilinson Medical

Center, Petah Tiqva,

and Sackler Faculty of

Medicine, Tel Aviv

University, Tel Aviv,

Israel

A Kuperman

P Merlob

Correspondence to:

M Snir, MD, Department of Ophthalmology, Beilinson

Medical Center, Petah Tikva, 49100, Israel.

Accepted for publication 11 March 1996
Bell's phenomenon (BP) was first described by Sir Charles Bell in 1823. He reported on upward deviation of the eye during attempted eyelid closure in the presence of a unilateral lower motor neuron defect of the seventh nerve. ${ }^{12}$ The innervational pathways controlling this complex of integrative reactions are not as yet clearly defined, but it is assumed that the centre of innervation includes the third and seventh nerve nuclei. ${ }^{12}$

$\operatorname{Cogan}^{3}$ investigated BP in 156 normal upwards and laterally, whereas in 24 a variable response was found. In addition, $\mathrm{Hall}^{4}$ and Hoyt $e t a l^{5}$ noted that transient disturbances of the vertical gaze are common in normal neonates. Francis and Loughhead ${ }^{6}$ studied this phenomenon in 508 randomly selected adult patients and found a wide range in the magnitude and nature of the deviation of the eye during Bell's reflex movement.

The purpose of the present study was to evaluate the presence of $\mathrm{BP}$ in premature babies, investigate its maturation, and compare these data with those found in full term newborns. volunteers; in 132, the eye deviated upwards or
Patients and methods

The study population included 69 randomly selected infants born in 1993 at Beilinson Medical Campus. The cohort consisted of two groups: group 1, 42 babies born between 37 and 41 weeks of gestation and group 2, 27 premature babies born between 28 and 36 weeks of gestation.

Exclusion criteria were: (1) caesarean section; (2) neurological disorders; (3) intraventricular haemorrhage; (4) hydrocephalus; (5) chromosomal abnormalities; (6) convulsions; (7) labour asphyxia; and (8) Apgar score less than 7 .

Birth weight, head circumference, medical and neurological status, including doll's eye reflex, pupillary light reflex, and oculovestibular reflex were recorded for each baby.

The BP evaluation covered the following factors: (a) opening the baby's eyelids wide while the baby was attempting to close them forcibly; (b) study of the eye movements while holding both eyelids open against resistance. The examination was repeated by a second observer. The ocular movements during Bell's reflex were graded as follows: 0 , no response; 1 , minimal response (pupil visible); 2, full response (globe rotates upwards and laterally or upwards only, while pupil is covered by the eyelid). The score for the ocular movements was defined as the Bell's phenomenon score (BPS).

The first ocular examination of the full term babies was performed on the third day of life. A second examination was performed at age 4 to 8 weeks on those babies who did not show BP or had a weak response on the first examination, and a third examination was performed at the age of 12 to 16 weeks on those babies who still did not show BP or had a weak response on the second examination. We evaluated the incidence of BP in the three consecutive examinations, as well as the correlation between the presence of BP and gestational age, sex, birth weight, and head circumference.

The 27 premature babies were divided into two age groups: (a) 28-33 weeks of gestation, 12 babies; (b) 34-36 weeks of gestation, 15 babies. All premature babies underwent three consecutive examinations; during the first week of life and 4 and 8 weeks post partum. We evaluated the presence of BP and the correlation of the Bell's response with gestational age, sex, and birth weight.

STATISTICAL ANALYSIS

In the full term group, the correlation between birth weight or head circumference and BP was evaluated by analysis of variance, and between 
Table 1 Bell's phenomenon score (BPS) on each examination in the full term babies

\begin{tabular}{lllllll}
\hline Examination (age) & $\begin{array}{l}\text { BPS-O } \\
(\%)\end{array}$ & $\begin{array}{l}\text { BPS-1 } \\
(\%)\end{array}$ & $\begin{array}{l}\text { BPS-2 } \\
(\%)\end{array}$ & Variations & $\begin{array}{l}\text { Lost to } \\
\text { follow up }\end{array}$ & Total \\
\hline 1st (3 days) & $21(50)$ & $6(14.3)$ & $12(28.5)$ & $3^{\star}$ & 0 & 42 \\
2nd (4-8 weeks) & $14(56)$ & $6(24)$ & $5(20)$ & 2 & 27 \\
3rd (9-16 weeks) & 0 & $1(6)$ & $16(94)$ & 3 & 20 \\
\hline
\end{tabular}

^Downward and inward direction.

BPS- $0=$ no response or unable to judge; $B P S-1=$ weak response, the pupil is still seen; BPS-2=full response, the globe rotates laterally, and the pupil is covered by the eyelid.

the gestational age and BP by the $\chi^{2}$ test. In the preterm group, the correlation between sex, gestational age, or birth weight and BP was evaluated by Student's $t$ test and the Fisher exact test.

\section{Results}

FULL TERM GROUP

Forty two neonates (27 males and 15 females) were included in the full term group. Their mean gestational age was 39.1 (SD 2.5) weeks (range 37-41weeks) and their mean birth weight, 3173.3 (785.8) g. The neurological examination, including doll's eye reflex, oculovestibular reflex, and pupillary light reflex of all 42 neonates was normal. In the first examination 21 of the 42 babies (50\%) showed no BP (BPS-0), six babies (14.3\%) showed a weak response (BPS-1), and only 12 babies $(28.5 \%)$ showed full BP (BPS-2). It should be pointed out that three babies $(7.2 \%)$ had an atypical movement consisting of downward and inward deviation (Table 1).

The second examination was performed on 25 of the 27 babies who showed a negative or weak Bell's response (two babies were lost to follow up). Of these, 14 babies (56\%) still did not present Bell's reflex by the age of 4 to 8 weeks. Six of the $25(24 \%)$ scored BPS-1, and only five babies (20\%) BPS-2. Altogether, by the age of 4-8 weeks, 20 out of 40 babies $(50 \%)$ showed BPS-2. The third examination included 17 of the 20 babies who had shown either a negative or weak BP on the second examination (three babies were lost to follow up). Sixteen (94\%) had BPS-2 by the age of 9 to 16 weeks and only one presented a weak response (BPS-1).

Table 2 Distribution of premature babies in birthweight groups

\begin{tabular}{lrcc}
\hline Weight & Males & Females & Total \\
\hline$<1500$ & 10 & 7 & 17 \\
$1501-2000$ & 3 & 3 & 6 \\
$2001-2500$ & 2 & 2 & 4 \\
Total & 15 & 12 & 27 \\
\hline
\end{tabular}

Table 3 Bell's phenomenon score (BPS) in each examination in the premature babies

\begin{tabular}{lllll}
\hline Examination (age) & BPS-O & BPS-1 & BPS-2 & Total \\
\hline 1st (1 week) & 27 & - & - & 27 \\
2nd (4 weeks) & 24 & - & $3^{\star}$ & 27 \\
3rd (8 weeks) & 19 & $1^{\star}$ & $4^{\star}$ & 24 \\
Total & 19 & 1 & 7 & \\
\hline
\end{tabular}

*Gestational age $34-36$ weeks.

BPS- $0=$ no response or unable to judge; BPS- $1=$ weak response the pupil is still seen; BPS-2=full response, the globe rotates laterally or upwards and laterally, and the pupil is covered by the eyelid.
In summary, 36 out of 37 babies (97.3\%) showed a fully developed BP (BPS-2) at the end of the follow up period 9 to 16 weeks post partum.

The correlation between the presence of a fully developed BP and the timing of the examination is presented in Figure 1.

We analysed the correlation between the presence of BPS-2 and birth weight, head circumference, and gestational age in the three subgroups of full term babies. No significant correlation was found between the mean birth weight of the three subgroups $(p=0.2)$, head circumference $(p=0.7)$, sex $(p=0.63)$, gestational age $(p=0.66)$, and the presence of BP.

\section{THE PRETERM GROUP}

Twenty seven premature babies (15 males and 12 females) were included in this group. Neurological evaluation, including doll's eye reflex, pupillary light reflex, and oculovestibular reflex was normal in all cases.

The gestational age ranged between 28 and 36 weeks, and their birth weight between 800 and $2500 \mathrm{~g}$ (Table 2, Fig 2). None of the premature babies showed a BP (BPS-0) during the first week of life, and only three of the $27(11.1 \%)$ presented a normal response (BPS-2) at 4 weeks. Only four of 24 babies (16.6\%) showed a fully developed BP (BPS-2) at the age of 8 weeks, and one baby $(4.16 \%)$ was graded as BPS- 1 .

In summary, only eight of the 27 premature babies $(29.6 \%)$ showed BPS-1 and 2 at the last examination at 8 weeks after birth (Table 3 ).

We evaluated the correlation between the presence of BPS and sex, gestational age, and birth weight. A statistically significant correlation was found between female sex and BPS incidence $(p=0.0248)$, as $78.9 \%(15 / 19)$ of the males and $21.1 \%(4 / 19)$ of the females had shown BPS-0.

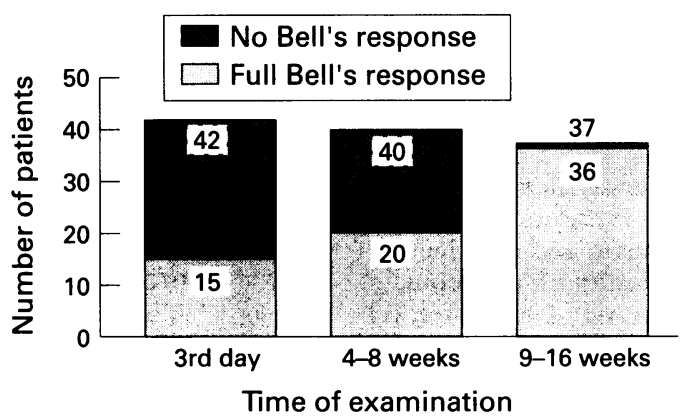

Figure 1 Frequency of appearance of Bell's phenomenon relative to postpartum age.

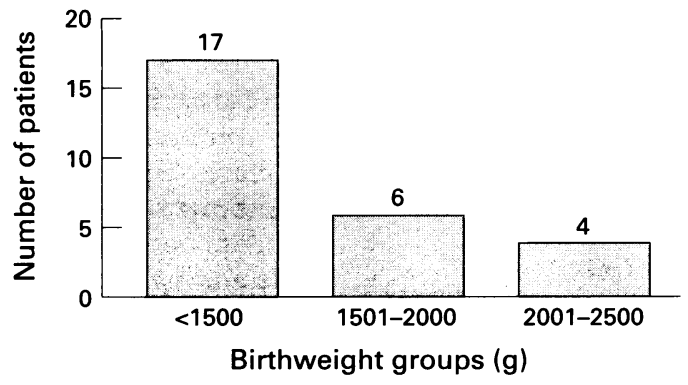

Figure 2 Number of preterm babies according to birth weight. 
However, in the BPS- 1 and 2 groups, $75 \%(6 / 8)$ were females and $25 \%(2 / 8)$ were males. A good correlation between birth weight and BPS was also demonstrated $(p=0.004)$. The birth weight of the premature babies with BPS- 0 ranged between 800 and $1400 \mathrm{~g}$ (mean 1117.9 (SD 213.2) g), and those with BPS-1 and 2, between 1550 and $2500 \mathrm{~g}$ (mean 1987.5 (SD 324.9) g). In addition, a highly significant correlation was found between babies with BPS-1 and 2 and gestational age $<0.0001)$. The mean age of the BPS-0 group was 31.4 (5.2) weeks, and the BPS-2 group was 35 (1.6) weeks.

\section{Discussion}

The precise neurological mechanism of Bell's reflex is not yet known, but it is clear that the seventh nerve nucleus in the pons and the third nerve nucleus in the rostral midbrain and the interconnecting pathways are part of the innervation complex. ${ }^{12}$

Ferrer ${ }^{7}$ studied the incidence of Bell's phenomenon among 379 normal children from birth to age 5. Up to the age of 2 months he found a normal upward gaze response in only 13 out of 124 babies (10.5\%). At the age of 4 months, $23.6 \%$ showed a normal Bell's reflex, and at the age of 8 months, $33.3 \%$. In addition, Ferrer ${ }^{7}$ examined a group of 180 children over 5 years of age and found a normal BP in $88.9 \%$.

Francis and Loughhead ${ }^{6}$ reported the presence of BP among 508 consecutive adult patients. Their study demonstrated a variability in the type of BP in $21 \%$ of the normal patients; none had a negative response, but $7 \%$ demonstrated only a minimal upwards response. In addition they found a frequency of $8 \%$ of patients with jownward responses, compared to the $3.7 \%$ reported by Ferrer. ${ }^{7}$

With regard to the full term babies in our study, $35.7 \%$ revealed a positive $\mathrm{BP}$ on the third day of life, $50 \%$ at age 4-8 weeks, and $97.3 \%$ in the fourth month of life. The discrepancy between the results of our study and of Ferrer's may be attributable to the significant difference in the number of examined babies and in the method of BP evaluation. Moreover, the variability in Bell's response and its clinical interpretation and the fact that repeated testing by two observers (as in our study) may give a higher positive yield, may also help to explain the different results.

Ferrer $^{7}$ also examined another group of $88 \mathrm{chil}-$ dren aged 8 to 14 years who were premature at birth and found a normal BP in $80.7 \%(71 / 88)$.
However, in $17 \%(15 / 88)$ the response was unusual, and the eyes deviated to one side. He defined this response as a 'spasm of conjugate gaze'. Unfortunately, Ferrer did not examine the presence of Bell's reflex in these preterm babies immediately after birth and, therefore, we cannot compare our results with his. It should be mentioned that in certain instances of parietal lobe lesions, the Bell's response is shifted laterally to the opposite side, probably due to spasticity of the conjugate gaze. ${ }^{38}$

In our data of the premature group (27 babies), BP was absent in the first week of life and at age 8 weeks only $29.6 \%(8 / 27)$ showed BPS-1 and 2 compared with the full term group in which $50 \%$ presented a mature BP.

These results demonstrate that $\mathrm{BP}$ has a longitudinal neurological development and is part of the normal neurological maturation process in both full term and premature babies.

It should be emphasised that the fully developed BP in the premature group was found only in those babies weighing more than $1500 \mathrm{~g}$ and with a mean gestational age of 35 (SD 1.6) weeks. Additionally, a good correlation was present in this subgroup between the BP and female sex, birth weight, and gestational age.

Our data support previous findings ${ }^{7}$ that BP is not commonly present in neonates in the first week of life. This gradual increase in the frequency of BP from premature to full term babies supports the assumption that maturity of the brain stem is a prerequisite for the normal appearance and the variability of this phenomenon. Even the findings of $\mathrm{Hall}^{4}$ and Hoyt et $a l^{5}$ support this assumption of the immaturity of brain stem reflexes in the perinatal period.

1 Duane TD. Duane's clinical ophthalmology. Vol 2. Philadelphia: JB Lippincott, 1991: Chapter 2, 22-3; Chapter 13, 6. Bell C. On the motions of the eye in illustration of the uses of the muscles and nerves of the orbit. Phil Trans $R$ Soc Lond 1823;113:166-86.

3 Cogan DG. Neurological significance of lateral conjugate deviation of the eyes on forced closure of the eyelids. Arch Ophthalmol 1948;39:37.

4 Hall AJ. Some observations on the acts of closing and opening the eyes. Br $\mathcal{O}$ Ophthalmol 1936;20:257-95.

5 Hoyt CS, Mousel DK, Weber AA. Transient supranuclear disturbances of gaze in healthy neonates. Am $\mathcal{F} O$ phthalmol 1980;89:708-13.

6 Francis IC, Loughhead JA. Bell's phenomenon. A study of 508 patients. Aust $\mathcal{f}$ Ophthalmol 1984;12:15-21.

7 Ferrer JA. Conclusions from Bell's phenomenon variants. Trans Am Acad Ophthalmol Otolaryngol 1973;77:714-20.

8 Smith JL, Gay AJ, Cogan DG. The spasticity of conjugate gaze phenomenon. Arch Ophthalmol 1959;62:694-6. 